\title{
Randomized Phase II study of the safety, efficacy and immune response of GVAX pancreas (with cyclophosphamide) and CRS-207 with or without nivolumab in patients with previously treated metastatic pancreatic adenocarcinoma (STELLAR)
}

\author{
Dung T Le ${ }^{1 *}$, Todd S Crocenzi ${ }^{2}$, Jennifer N Urum³ ${ }^{3}$, Eric R Lutz', Daniel A Laheru ${ }^{3}$, Elizabeth A Sugar ${ }^{4}$, \\ Robert H Vonderheide ${ }^{5}$, George A Fisher ${ }^{6}$, Andrew $\mathrm{H} \mathrm{Ko}^{7}$, Aimee L Murphy ${ }^{8}$, Katherine McDougall', \\ Sandy Ferber ${ }^{10}$, Dirk G Brockstedt ${ }^{11}$, Elizabeth M Jaffee ${ }^{1}$ \\ From 30th Annual Meeting and Associated Programs of the Society for Immunotherapy of Cancer (SITC \\ 2015) \\ National Harbor, MD, USA. 4-8 November 2015
}

\section{Background}

A heterologous prime-boost vaccination strategy using GVAX pancreas vaccine and CRS-207 is showing promise in patients with pancreatic adenocarcinoma (PDA) (Le, JCO 2015). Furthermore, blockade of the immune checkpoint programmed death-1 (PD-1) is active in some cancers. Combinatorial strategies aimed at priming tumor antigen-specific $\mathrm{T}$ cells while simultaneously blocking negative checkpoints may be necessary to improve outcomes in PDA. GVAX is composed of allogeneic pancreatic cancer cells modified to express GM-CSF and induces a broad response against multiple tumor antigens. GVAX is given with low-dose cyclophosphamide (CY) to inhibit regulatory T cells. CRS-207 is live-attenuated Listeria monocytogenes engineered to express the tumor-associated antigen mesothelin. CRS-207 boosts responses against mesothelin and is unique in its capacity to stimulate both innate and adaptive immunity by activating $\mathrm{T}$ cells and NK cells. Nivolumab is an antibody against PD-1.

\section{Methods}

This is a Phase II study comparing CY/GVAX and CRS207 with or without nivolumab in subjects with PDA who

\footnotetext{
${ }^{1}$ The Sidney Kimmel Comprehensive Cancer Center at Johns Hopkins, Baltimore, MD, USA

Full list of author information is available at the end of the article
}

failed only one chemotherapy regimen for metastatic disease. Subjects are randomized in a 1:1 ratio to receive either 2 doses of CY/nivolumab/GVAX and 4 doses of nivolumab/CRS-207 (Arm A) or 2 doses of CY/GVAX and 4 doses of CRS-207 (Arm B). The primary objective is to compare OS between Arms A and B. Secondary/ exploratory objectives include: assessment of safety and clinical responses (tumor assessments and CA19-9 levels) and correlation of $L m$ - and mesothelin-specific $\mathrm{T}$ cell and other immunological responses with OS, progression-free survival and best overall response.

\section{Authors' details}

${ }^{1}$ The Sidney Kimmel Comprehensive Cancer Center at Johns Hopkins, Baltimore, MD, USA. ${ }^{2}$ Providence Cancer Center, Portland, OR, USA. ${ }^{3}$ The Sidney Kimmel Comprehensive Cancer Center at Johns Hopkins, Baltimore, MD, USA. ${ }^{4}$ Departments of Biostatistics and Epidemiology, Johns Hopkins Bloomberg School of Public Health, Baltimore, MD, USA. ${ }^{5}$ University of Pennsylvania, Baltimore, MD, USA. 'Stanford University School of Medicine, Stanford, CA, USA. ${ }^{7}$ UCSF Helen Diller Family Comprehensive Cancer Center, San Francisco, CA, USA. ${ }^{8}$ Aduro BioTech, Inc., Berkeley, CA, USA. ${ }^{9}$ Aduro Biotech, Berkeley, CA, USA. ${ }^{10}$ Array Biostatistics LLC, Evanston, IL, USA.

${ }^{11}$ Aduro Biotech, Inc., Berkeley, CA, USA.

Published: 4 November 2015

doi:10.1186/2051-1426-3-S2-P155

Cite this article as: Le et al:: Randomized Phase II study of the safety, efficacy and immune response of GVAX pancreas (with cyclophosphamide) and CRS-207 with or without nivolumab in patients with previously treated metastatic pancreatic adenocarcinoma (STELLAR). Journal for ImmunoTherapy of Cancer 2015 3(Suppl 2):P155. 